\title{
Video-assisted thoracoscopic bilateral lung metastasectomy with a subxiphoid access port
}

\author{
Yuji Taniguchi, MD, ${ }^{\text {a }}$ Yoshimasa Suzuki, MD, ${ }^{\mathrm{b}}$ Takako Suda, MD, ${ }^{\mathrm{b}}$ \\ Tomoko Inoue, MD, ${ }^{\mathrm{b}}$ Kunio Araki, MD, ${ }^{\mathrm{b}}$ Norimasa Ito, MD, ${ }^{\mathrm{b}}$ \\ Koichiro Okada, MD, ${ }^{\mathrm{b}}$ Kiyosuke Ishiguro, MD, ${ }^{\mathrm{b}}$ Hiroshige Nakamura, MD, ${ }^{\mathrm{a}}$ and \\ Shigetsugu Ohgi, MD, ${ }^{\mathrm{b}}$ Yonago, Japan
}

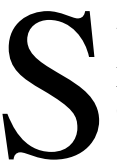

urgical treatment of tumor lesions in the bilateral lung is performed by a bilateral thoracotomy, a median sternotomy, and a clamshell incision. Recently, thoracoscopic surgery has become widely used for the treatment of metastatic lesions in the bilateral lung. ${ }^{1}$ Because conventional thoracoscopic surgery for the treatment of metastatic lesions in the bilateral lung is performed in the lateral decubitus position, changes in body position are troublesome. We developed a thoracoscopic partial resection of the bilateral lung with metastatic lesions using an access port inserted below the xiphoid process for which changes in the body position are not required.

\section{Clinical Summary}

A 72-year-old man was admitted to our hospital because of metastatic lung cancer after right hemicolectomy for ascending colon cancer. Chest radiography demonstrated three tumor shadows in the left upper lung field. Chest computed tomography revealed three tumor shadows in the left lung of S1+2a, S1+2c, S3a, and $\mathrm{S} 3 \mathrm{~b}$ and one tumor shadow in the right lung of S5b (Figure 1). We performed thoracoscopic surgery.

The operation was performed in the supine position after achievement of general anesthesia with a double-lumen endobronchial tube. The right lung was treated first, followed by the left lung. Thoracoports were inserted in the fourth and sixth intercostal anterior axillary lines on the right side and the third and fifth intercostal anterior axillary lines on the left side. An access port below the xiphoid process was prepared by the following procedures. Skin, with a length of $2.5 \mathrm{~cm}$, was incised from the xiphoid process to the abdominal median, and the xiphoid process was exposed by incision of the upper white line. Exfoliation was performed along the substerunum from the xiphoid process. A port with a shaft length of $109 \mathrm{~mm}$ (Core Dynamics Entree II R; Conmed Co, New York, NY) was inserted into the thoracic cavity.

\footnotetext{
From the Department of General Thoracic Surgery, ${ }^{a}$ Tottori University Hospital, and the Division of Organ Regeneration Surgery, ${ }^{\text {b Faculty of }}$ Medicine, Tottori University, Yonago, Japan.

Received for publication Feb 1, 2005; accepted for publication March 1, 2005 .

Address for reprints: Yuji Taniguchi, MD, Department of General Thoracic Surgery, Tottori University Hospital, 36-1 Nishi-Cho, Yonago, Tottori, 683-8504 Japan (E-mail: kuichi@grape.med.tottori-u.ac.jp).

J Thorac Cardiovasc Surg 2005;130:916-7

$0022-5223 / \$ 30.00$

Copyright (C) 2005 by The American Association for Thoracic Surgery

doi:10.1016/j.jtcvs.2005.03.039
}

The costal arch for the operation was lifted

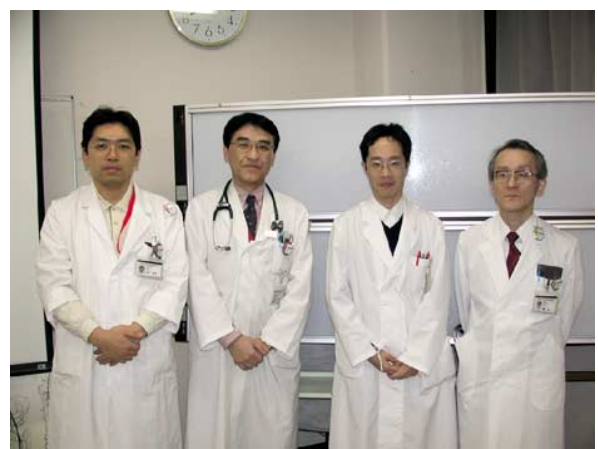

Taniguchi, Nakamura, Ito, Ohgi (left to right) by using a lifting apparatus with a subcutaneously inserted $1.5-\mathrm{mm}$ wire (Wall-lifting with subcutaneous wiring set; Mizuho Co, Tokyo, Japan; Figure 2). In both the right and left thoracic cavities, rough adhesion was observed in wide areas, but exfoliation of the adhesion was completed in each cavity by means of operation from three ports alone, including the subxiphoid access port. No lesions other than those detected by preoperative chest computed tomography were observed in the right and left thoracic cavities by thoracoscopy or palpation with Thoraco Cotton (Kenzmedico Co, Saitama, Japan). Metastasectomy was performed for the removal of one tumor lesion in the right lung and four tumor lesions in the left lung. Insertion of the thoracoscope and the automatic suture apparatus was performed through the ports indwelled between the ribs and the subxiphoid access port. The amount of hemorrhage during the operation was $5 \mathrm{~g}$. The postoperative course was good and without complications.

\section{Discussion}

It is generally necessary to change the body position to the lateral decubitus position to perform bilateral lung resection without sternotomy. Changes in body position are troublesome. However, in thoracoscopic partial resection of the bilateral lungs with a subxiphoid access port, changes in body position and sternotomy are not required. Furthermore, the method can be easily converted to a median sternotomy or hand-assisted thoracoscopic surgery (HATS) if necessary.

Mineo, ${ }^{2}$ Utsumi, ${ }^{3}$ and their colleagues reported methods for reaching the bilateral thoracic cavities from a site below the xiphoid process, but both methods were HATS. It was reported that the circulatory dynamics became unstable because of insertion of a hand into the thoracic cavity. ${ }^{3}$

We were concerned about difficulty in the operation on lesions on the dorsal side of the left lower lobe and the effects of pressure on the heart on the circulatory dynamics in this method, as in HATS. ${ }^{3}$ However, because the most dorsal lesion in this patient was at the left $\mathrm{S} 1+2 \mathrm{c}$ in the upper lobe and all lesions were in the peripheral areas, we performed surgical intervention through this method and obtained a good outcome. In this patient the operation with devices inserted from the subxiphoid access port was improved by lifting the costal arch with wire. Furthermore, there was 

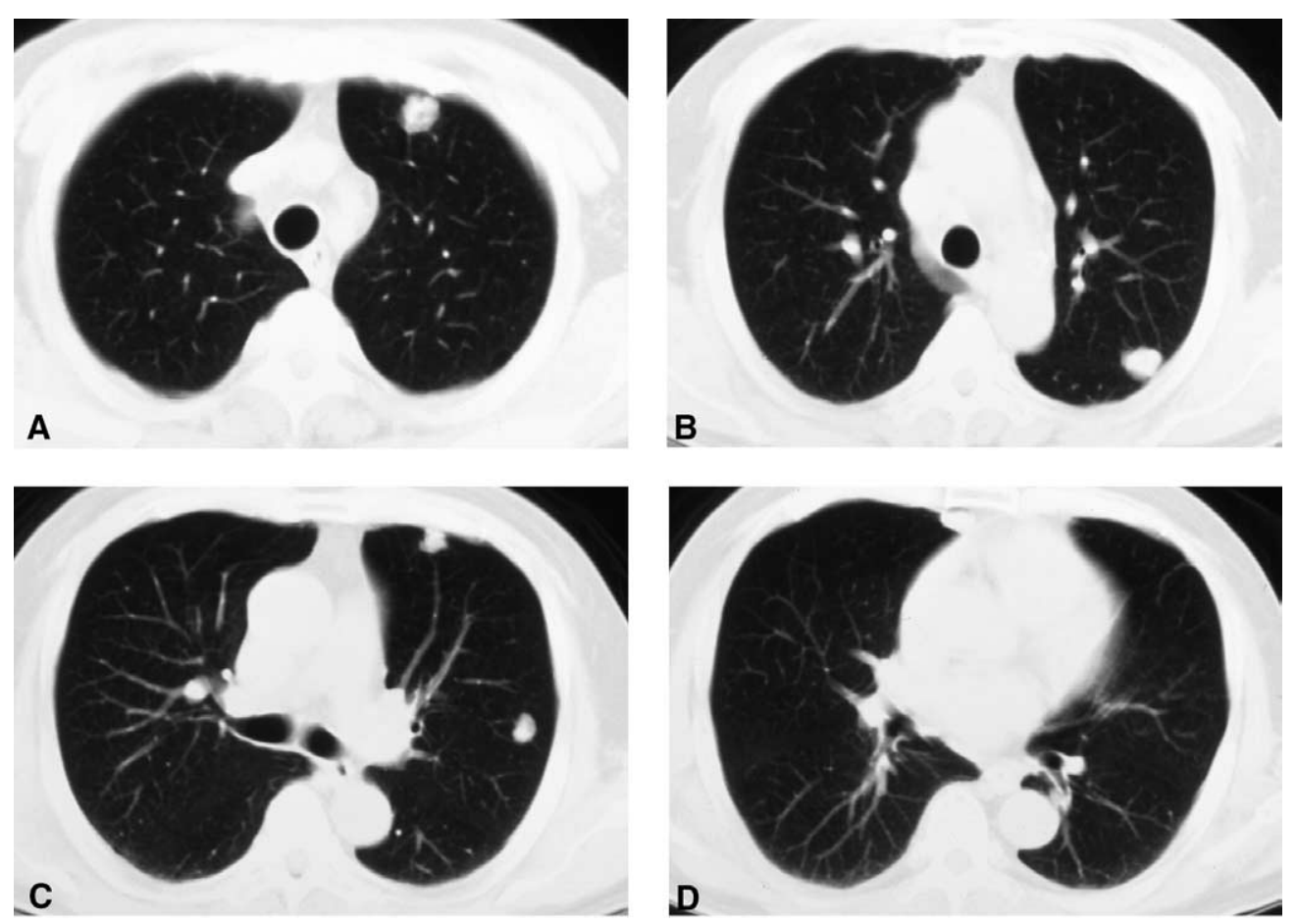

Figure 1. Chest computed tomography showed 5 metastatic lesions. A, left S1+2a. B, Left S1+2c. C, Left S3a and S3b. D, Right S5b.

almost no pressure on the heart, and the circulatory dynamics were stable during the operation.

Thoracoscopic partial resection of the bilateral lungs with a subxiphoid access port might be a useful method for the surgical treatment of benign diseases and metastatic tumor in the bilateral lungs. However, problems remain to be solved in the future, such as further technical improvement of the treatment of lesions in the bilateral dorsal area and evaluation of the application of conversion to HATS.

\section{References}

1. Dowling RD, Keenann RJ, Ferson PF, Landreneau RJ. Video-assisted thoracoscopic resection of pulmonary metastases. Ann Thorac Surg. 1993;56:772-5.

2. Mineo TC, Pompeo E, Ambrogi V, et al. Video-assisted approach for transxiphoid bilateral lung metastasectomy. Ann Thorac Surg. 1999;67: 1808-10.

3. Utsumi T, Kido T, Yasukawa M, et al. Hand-assisted thoracoscopic surgery (HATS) [in Japanese with English abstract]. Jpn J Chest Surg. 2002;16:117-20.
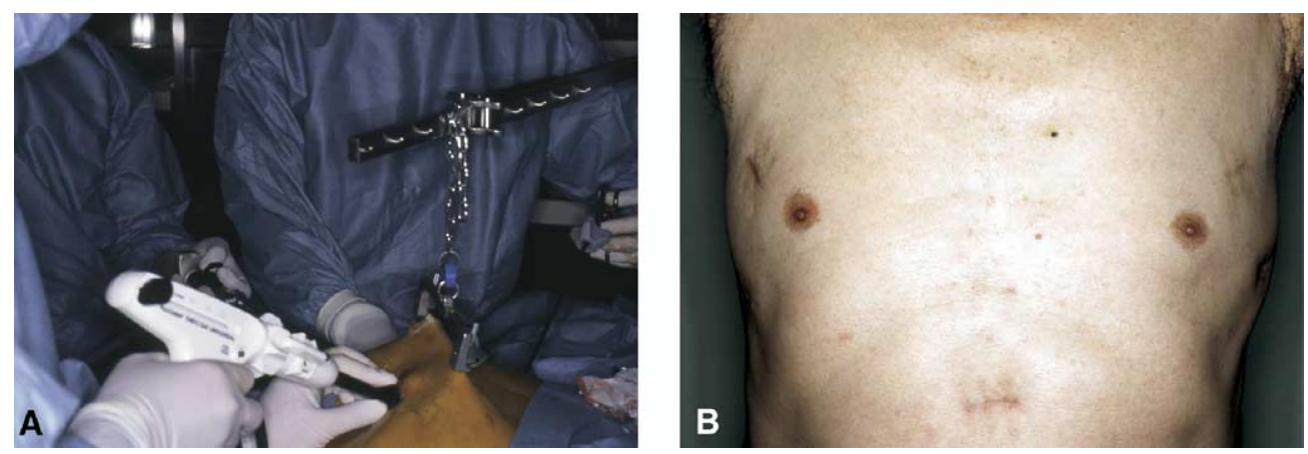

Figure 2. A, Thoracoscopic metastasectomy by using a subxiphoid access port with lifting the costal arch. B, View of the postoperative wound. 